\title{
Global Structure of Mirror Modes in the Magnetosheath
}

\author{
Jay R. Johnson and C. Z. Cheng \\ Princeton Plasma Physics Laboratory, Princeton, NJ 08543
}

\begin{abstract}
A global stability analysis of mirror modes in the magnetosheath is presented. The analysis is based upon the kinetic-MHD formulation which includes relevant kinetic effects such as Landau resonance and gradient drift effects related to inhomogeneities in the background density, temperature, pressure and its anisotropy, magnetic field, and plasma flow velocity. Pressure anisotropy provides the free energy for the global mirror mode. The local theory of mirror modes predicts purely growing modes confined in the unstable magnetosheath region; however, the nonlocal theory that includes the effects of gradients and plasma flow predicts modes with real frequencies which propagate with the flow from the magnetosheath toward the magnetopause boundary. The real frequency is on the order of a combination of the diamagnetic drift frequency and the Doppler shift frequency associated with plasma flow. The diamagnetic drift frequency provides a wave phase velocity in the direction of the magnetopause so that wave energy accumulates against the magnetopause boundary, and the amplitude is skewed in that direction. On the other hand, plasma flow also gives rise to a real phase velocity, but the phase velocity is smaller than the flow velocity. As a result, the wave amplitude is increased in the wake of the plasma flow and piles up against the bow shock boundary.
\end{abstract}

DISTRIBUTION OF THIS DOCUMENT IS UNLIMITED 


\section{DISCLAIMER}

This report was prepared as an account of work sponsored by an agency of the United States Government. Neither the United States Government nor any agency thereof, nor any of their employees, make any warranty, express or implied, or assumes any legal liability or responsibility for the accuracy, completeness, or usefulness of any information, apparatus, product, or process disclosed, or represents that its use would not infringe privately owned rights. Reference herein to any specific commercial product, process, or service by trade name, trademark, manufacturer, or otherwise does not necessarily constitute or imply its endorsement, recommendation, or favoring by the United States Government or any agency thereof. The views and opinions of authors expressed herein do not necessarily state or reflect those of the United States Government or any agency thereof. 


\section{DISCLAIMER}

Portions of this document may be illegible in electronic image products. Images are produced from the best available original document. 


\section{INTRODUCTION}

Recently much attention has been given to the identification of wave modes in the magnetosheath and, in particular, near the magnetopause [1-10]. Such efforts are of interest primarily because the identified modes may grow to substantial amplitudes and can cause significant change in plasma profiles and pressure anisotropy. For example, it is thought that ion-cyclotron waves control the degree of pressure anisotropy in the magnetosheath [11]. Moreover, low frequency MHD waves such as mirror modes excited in the magnetosheath or fast compressional Alfvén waves originating from the bow shock can propagate to the magnetopause and lead to significant plasma transport. Transport at the magnetopause due to low frequency MHD wave turbulence can be very efficient because magnetic field and density gradients at the magnetopause efficiently couple large scale wave energy into small scale kinetic Alfvén waves with perpendicular wavelength on the order of the gyroradius [12-15]. Using quasilinear theory we have shown that these waves can lead to efficient particle transport across the magnetic field for both northward and southward IMF. Moreover, for southward IMF, we have shown that kinetic Alfvén waves can propagate to the location because the Landau damping is suppressed by magnetic gradient and curvature drifts [14]. As a consequence, magnetic islands can form and for a typical spectrum of low frequency $\mathrm{MHD}$ waves, there will be multiple overlapping islands in the particle phase space which can lead to massive particle transport.

The mirror modes destabilized in the magnetosheath are of substantial interest because they can couple a significant amount of wave energy from the magnetosheath to waves at the magnetopause. The mirror modes are unstable and can grow to large amplitudes because the magnetosheath is characterized by a large pressure anisotropy [16,17]. Based on local mirror mode theory the mirror modes is purely growing and has no real frequency so as a consequence they would be confined in the magnetosheath and can not propagate to the magnetopause. In this work we shall study the nonlocal effects of realistic background gradients and plasma flow and demonstrate that the mode frequency is actually complex with 
a substantial real component due to particle diamagnetic drift and Doppler shift associated with plasma flow. With a substantial real frequency, these modes can propagate to the magnetopause and efficiently mode convert into kinetic Alfvén waves which may play an important role in transport processes at the magnetopause.

Large pressure anisotropy develops in the magnetosheath because as plasma crosses the bow shock, the magnetic field increases and the flow velocity perpendicular to the magnetic field is converted into gyromotion yielding a large perpendicular plasma temperature. Motion along the magnetic field is not readily converted into thermal energy so that a large anisotropy develops with $\beta_{\perp}>\beta_{\|}$. In the magnetosheath the kinetic pressure dominates the relatively weak magnetic field so that typically $\beta>1$. For $\beta_{\perp} / \beta_{\|}>\left(1+1 / \beta_{\perp}\right)$ the plasma is unstable to the well known mirror mode. Toward the magnetopause, the flow slows down and the magnetic field further increases while the plasma pressure decreases and becomes more isotropic. The plasma $\beta$ falls to a more typical magnetospheric value much less than 1 which suppresses the mirror mode instability.

Kinetic effects on mirror modes in the magnetosheath have been extensively studied using Vlasov theory based upon a homogeneous background $[3,9,11,18-20]$. While these calculations provide very useful information about local threshold conditions, they do not account for kinetic effects that involve background gradients and boundary conditions. On the other hand, MHD wave analysis effectively deals with complicated boundary conditions and global gradients, but fails to account for important kinetic effects.

While one- and two-dimensional hybrid simulations $[10,21]$ have the advantage of simulating many ion kinetic effects, it is difficult to prescribe and maintain a realistic steady state magnetic field geometry with appropriate boundary conditions. We employ the kinetic-MHD approach which is an attempt to incorporate the most important ion kinetic effects into the MHD formalism $[22,23]$. The gyrokinetic equation, which is essentially the gyroaveraged Vlasov equation, is the cornerstone of the kinetic part of the model for this application. Moments of the gyrokinetic equation are taken and provide a modified momentum equation and Ohm's law. This approach retains the important kinetic effects of Landau damping and 
diamagnetic drift as well as the physics associated with the ion magnetic gradient and curvature drifts. Moreover, it is straightforward to generalize this model to account for realistic two or three dimensional equilibrium profiles.

The organization of this paper is as follows. We shall briefly review the mirror mode and point out the important consequences introduced by (a) background gradients in the equilibrium and (b) kinetic effects. We present the kinetic-MHD model for the mirror mode which includes these effects. Then, we solve for the global mirror eigenmodes using a onedimensional equilibrium based upon typical observations and interpret the results. Then we will present a discussion of our results in relation to wave observations and simulation results. Finally, we give a summary.

\section{BACKGROUND GRADIENT AND KINETIC EFFECTS ON MIRROR MODES}

Without kinetic effects, low-frequency $\left(\omega \ll k_{\|} v_{A}, k_{\|} c_{s}\right.$, where $k_{\|}$is the parallel wave number, $v_{A}$ is the Alfvén speed, and $c_{s}$ is the sound speed) compressional magnetic field fluctuations in a plasma with flow velocity $V_{0}$ may be described by the MHD equation

$$
\left(\frac{\partial}{\partial t}+\mathbf{V}_{0} \cdot \nabla\right)^{2} \mathbf{B} \cdot \delta \mathbf{B}=\mathbf{B} \cdot \nabla\left(\frac{\sigma}{B^{2}} \mathbf{B} \cdot \nabla \mathbf{B} \cdot \delta \mathbf{B}\right)+\nabla_{\perp}^{2}\left(\tau_{M H D} \mathbf{B} \cdot \delta \mathbf{B}\right)
$$

where the firehose and mirror instability parameters are respectively

$$
\sigma=1+\left(\beta_{\perp}-\beta_{\|}\right) / 2
$$

and

$$
\tau_{M H D}=1+\beta_{\perp}\left(1-\beta_{\perp} / \beta_{\|}\right)
$$

for bi-Maxwellian plasmas. The uniform plasma dispersion relation for waves without background flow is $\omega^{2}=\sigma k_{\|}^{2} v_{A}^{2}+\tau_{M H D} k_{\perp}^{2} v_{A}^{2}$ which reduces in the isotropic limit $(\sigma=\tau=1)$ to the well known compressional Alfvén wave. In a plasma with $\beta_{\perp}>\beta_{\|} \sim 1, \tau_{M H D}<0$ can lead to the well known purely growing mirror instability. 
Modifications to the simplistic uniform plasma MHD description of the compressional wave can be significant. In the magnetosheath and near the magnetopause it is important to consider the effects of gradients in the equilibrium as well as kinetic effects. The parameter, $\tau_{M H D}$, which indicates regions of local instability when negative, has a profile which changes from positive in the solar wind (relatively isotropic pressure) to negative in the magnetosheath (due to large pressure anisotropy) to positive in the magnetosphere (low $\beta$, less anisotropy). As a consequence, (1) describes a purely growing eigenmode localized in the region of instability. In the presence of uniform background plasma flow, the mirror mode is purely growing in the plasma frame and should be convected with the flow. However, if the background plasma flow is nonuniform, mirror modes can acquire a finite phase velocity due to Doppler shift. The wave phase velocity roughly equals an average flow velocity in the unstable region. As the plasma flow slows down toward the magnetopause, wave energy pile up depends on the wave phase velocity with respect to the plasma flow velocity.

If kinetic effects are included in Eq. (1), kinetic contributions arise which modify the term proportional to $\tau_{M H D}$ so that $\tau_{M H D}$ must be replaced by an appropriate kinetic $\tau$ which contains effects of diamagnetic drift, wave-particle resonances and finite Larmor radii. In the low frequency limit $\left(\omega \ll k_{\|} v_{t h \|}\right)$, the kinetic $\tau$ reduces to $\tau_{M H D}$. It has already been shown that kinetic effects strongly affect the MHD picture of the mirror instability. If ( $\left.\omega \sim k_{\|} v_{t h \|}\right)$, then wave particle resonance is important. Hasegawa [24] first provided a description of kinetic effects on the mirror mode. More recently extensive studies of local mirror mode theories including kinetic effects have been compiled [1,3,9,11,18-20]. Southwood [25] provided an excellent physical picture of the kinetic effects on the instability and relates the instability to resonant particles with zero phase velocity. An important feature of the kinetic dispersion relation is that for an anisotropic plasma, in addition to the weakly damped fast magnetosonic wave found in the limit $\omega \gg k_{\|} v_{t h \|}$, the mirror mode is found with $\omega \ll k_{\|} v_{t h \|}$. For low frequencies $\left(\omega \ll k_{\|} v_{t h \|}\right)$, the kinetic mirror mode is reasonably approximated by the MHD description for the threshold condition. However, when $\omega \sim k_{\|} v_{t h \|}$, the kinetic mirror mode growth rates are significantly reduced from the prediction of MHD theory. 
Other kinetic effects not described by uniform plasma Vlasov theory also arise where pressure and density gradients are important. Hasegawa first described these effects as the drift-mirror mode [24], but these effects were thought to be unimportant because pressure and density gradients in the magnetosheath are generally small; however, we shall show that large localized gradients at the magnetopause can affect the global structure and provide a real frequency to the mirror mode. The gradients enter into the kinetic description through the diamagnetic drift. At the magnetopause, the diamagnetic drift frequency, $\omega_{*} \sim k_{\perp} v_{t h i} \rho_{i} / L_{M P}\left(L_{M P}\right.$ is the gradient scale at the magnetopause and $\rho_{i}$ is the ion gyroradius) can be significant because, for typical magnetopause parameters, $\omega_{*} \sim k_{\|} v_{t h i}$ and wave-particle interaction is strongly modified.

\section{KINETIC-MHD EIGENMODE EQUATION FOR MIRROR MODES}

An appropriate description of the mirror mode in the magnetosheath and near the magnetopause should attempt to account for the effects outlined in the last section. In particular, the model should contain the global profile effects which arise for inhomogeneous flow velocity, density, pressure, temperature, and magnetic field. Moreover, the model should contain important kinetic effects associated with the wave-particle interaction and diamagnetic drift. The gyrokinetic-MHD model, which retains all of these effects is appropriate because the smallest background scale (the magnetopause) is typically the order of $10 \rho_{i}$ and thus satisfies the gyrokinetic approximation. To focus on the effects described above, we take for simplicity a one-dimensional equilibrium in the $x$ direction which is taken to be normal to the magnetopause. While we expect that the two-dimensional and three-dimensional nature of the magnetopause can be important, the one-dimensional equilibrium gives us an abundance of new insight on the mirror mode. All equilibrium quantities will be functions of $x$ only. For simplicity, we take the magnetic field and velocity to be $B_{0}(x) \hat{z}$ and $V_{0}(x) \hat{x}$ respectively. We neglect drifts due to magnetic field gradients and curvature in the resonant wave-particle interaction (which at most will shift the real frequency of the modes by a negligible amount). 
Coupling to transverse magnetic fluctuations is ignored. If $\omega<k_{\|} c_{s}, k_{\|} v_{A}$ this assumption is reasonable, but we expect that there will be localized coupling near the location where the phase velocity of the wave approaches $v_{A}$ or $c_{s}$ leading to mode conversion to kinetic Alfvén waves or damping from the sound continuum.

The global kinetic-MHD equation describing the compressional magnetic field is given by

$$
\left(\frac{\partial}{\partial t}+\mathbf{V}_{0} \cdot \nabla\right)^{2} \mathbf{B} \cdot \delta \mathbf{B}=\mathbf{B} \cdot \nabla\left(\frac{\sigma}{B^{2}} \mathbf{B} \cdot \nabla \mathbf{B} \cdot \delta \mathbf{B}\right)+\nabla_{\perp}^{2}(\tau \mathbf{B} \cdot \delta \mathbf{B})
$$

where

$$
\tau \mathbf{B} \cdot \delta \mathbf{B} \equiv \tau_{M H D} \mathbf{B} \cdot \delta \mathbf{B}+\delta \hat{p}_{\perp}
$$

which arise through the nonadiabatic pressure response,

$$
\delta \hat{p}_{\perp}=m \int d^{3} v\left(\mathrm{v}_{\perp}-\mathrm{V}_{\mathbf{0}}\right)^{2} g / 2
$$

The nonadiabatic particle distribution, $g$, evolves according to the gyrokinetic equation which is given by

$$
\left(\omega-i \mathbf{v}_{\|} \cdot \nabla-i \mathbf{V}_{0} \cdot \nabla\right) g=-\frac{q}{m} \frac{\partial F}{\partial \mathcal{E}}\left(1-\frac{\omega_{*}}{\omega}\right) \frac{\omega v_{\perp}}{k_{\perp}} J_{1}\left(k_{\perp} v_{\perp} / \Omega\right) \frac{\mathbf{B} \cdot \delta \mathbf{B}}{B}
$$

where $\mathcal{E}=v^{2} / 2, \Omega$ is the cyclotron frequency, and $\omega_{*}=\mathrm{B} \times \mathrm{k}_{\perp} \cdot \nabla F /(B \Omega \partial F / \partial \mathcal{E})$. It is implicitly understood that $k_{\perp}$ and $\omega_{*}$ are operators [26]. In Eq.(7) we have dropped terms related to magnetic gradient and curvature drifts. In deriving Eq. (4) we have also dropped terms which couple to the transverse magnetic field through the pressure gradient and magnetic field curvature. However, Eq. (4) contains the essential kinetic effects required to replicate the well known drift mirror mode dispersion relation [24]. Because $k_{\perp} \rho_{i}<1$ we may formally expand the Bessel function to retain nonlocal effects in Eq. (7). We solve the gyrokinetic equation using an expansion equivalent to a WKB approximation in the $\hat{\mathbf{x}}$ direction. We verify the validity of that approximation a posteriori. This approximation allows us to replace $\mathbf{V}_{0} \cdot \nabla g$ by $g \mathbf{V}_{0} \cdot \nabla \delta B_{\|} / \delta B_{\|}$. From Eq. (6) we obtain 


$$
\begin{gathered}
\delta \hat{p}=-\frac{\beta_{\perp} T_{\perp}}{T_{\|}}\left[\left(\zeta-\zeta_{*}^{(1)}\right) Z(\zeta) 2 \mathcal{I}_{3} / \lambda-\zeta_{*}^{(2)} Z(\zeta) 2 \mathcal{I}_{5} / \lambda+\right. \\
\left.\zeta_{*}^{(3)} \zeta(1+\zeta Z(\zeta)) 2 \mathcal{I}_{3} / \lambda\right] \mathbf{B} \cdot \delta \mathbf{B} \\
\zeta=\frac{\left(\omega-i V_{0} \cdot \nabla\right)}{\sqrt{2} k_{\|} v_{t h \|}} \\
\zeta_{*}^{(1)}=\frac{\mathbf{b} \times \mathbf{k}_{\perp} v_{t h \perp}}{\sqrt{2} k_{\|} v_{t h \|}} \rho_{i}\left(\frac{\nabla n}{n}+\frac{1}{2} \frac{\nabla T_{\|}}{T_{\|}}-\frac{\nabla T_{\perp}}{T_{\perp}}\right) \\
\zeta_{*}^{(2)}=\frac{\mathbf{b} \times \mathbf{k}_{\perp} v_{t h \perp}}{\sqrt{2} k_{\|} v_{t h \|}} \rho_{i}\left(\frac{\nabla T_{\perp}}{T_{\perp}}\right) \\
\zeta_{*}^{(3)}=\frac{\mathbf{b} \times \mathbf{k}_{\perp} v_{t h \|}}{\sqrt{2} k_{\|} v_{t h \|}} \rho_{i}\left(\frac{\nabla T_{\|}}{T_{\|}}\right)
\end{gathered}
$$

where $\lambda=k_{\perp}^{2} \rho_{i}^{2} / 2$. The integral operators, $\mathcal{I}_{3}, \mathcal{I}_{5}$ are defined in the Appendix and involve integrals over Bessel's functions. For small $k_{\perp} \rho_{i}, 2 \mathcal{I}_{3} / \lambda \sim 1$ and $2 \mathcal{I}_{5} / \lambda \sim 3$.

Boundary conditions at the bow shock and magnetopause are critical in determining the structure of the modes. Because the group velocity of the modes that we consider are far less than the flow velocity in the solar wind, no wave information can be carried across the bow shock. This property is manifest in a singularity at the location where $\tau_{M H D}=\left(V_{0} / v_{A}\right)^{2}=M_{A}^{2}$. At that location, the group velocity of compressional waves propagating against the flow approaches zero. A careful expansion of the MHD equations near this point indicates that the solution can consist of two Frobenius solutions, one of which is a power series and the other dominated by a logarithmic singularity. The behavior of the solutions is very similar to the behavior of MHD solutions near the well known field line resonance [27]. In this case, however, we choose the coefficient of the logarithmic solution to vanish because there is no physical mechanism which can provide the buildup of energy at the boundary. The remaining solution behaves like $c\left(1+\eta^{2} x+\ldots\right)$ where $\eta=\omega / \nabla V_{0}$ with $\eta \ll 1$ for a sharp boundary. In essence, this means that $d \delta B_{\|} / d x \approx 0$ near the boundary which can be interpreted as a reflecting boundary condition. The amplitude of the wave 
should reduce to zero in a small boundary layer near the bow shock when appropriate kinetic effects and coupling are include. We take the approximation $d \delta B_{\|} / d x \approx \eta^{2}$ near the bow shock in all of our results. On the magnetospheric side of the magnetopause we take the boundary condition that the solution connects to the appropriate exponentially decaying Eikonal solution which we obtain implicitly from the differential equation.

\section{LOCAL THEORY}

To get a better understanding of the physical meaning of the kinetic-MHD eigenmode equation we can investigate local theory with a WKB representation, $\nabla \rightarrow i \mathrm{k}=i \mathrm{k}_{\perp}+i k_{\|} \mathrm{b}$, and small $k_{\perp} \rho_{i}$. The local dispersion relation becomes

$$
\left(\omega-\mathrm{k} \cdot \mathrm{V}_{0}\right)^{2}=\sigma k_{\|}^{2} v_{A}^{2}+\tau k_{\perp}^{2} v_{A}^{2}
$$

where

$$
\begin{aligned}
\tau=1 & +\beta_{\perp}\left\{1-\beta_{\perp} / \beta_{\|}\left[1-\left(\zeta-\zeta_{*}^{(1)} Z(\zeta) 2 \mathcal{I}_{3} / \lambda\right.\right.\right. \\
& \left.\left.\left.-\zeta_{*}^{(2)} Z(\zeta) 2 \mathcal{I}_{5} / \lambda+\zeta_{*}^{(3)} \zeta(1+\zeta Z(\zeta)) 2 \mathcal{I}_{3} / \lambda\right)\right]\right\}
\end{aligned}
$$

From the appropriate limits it is not difficult to see that this dispersion relation governs

propagation of compressional Alfvén (fast magnetosonic) waves for $\left(\omega-\mathrm{k} \cdot \mathrm{V}_{0}\right)>k_{\|} v_{t h i}$ and the mirror mode for $\left(\omega-\mathbf{k} \cdot V_{0}\right)<k_{\|} v_{t h i}$. Without diamagnetic drift effects, the frequency and damping rate of the weakly damped magnetosonic wave are given by

$$
\begin{array}{r}
\omega_{r} \approx \mathrm{k} \cdot \mathrm{V}_{0} \pm\left[\sigma k_{\|}^{2}+\left(1+\beta_{\perp}\right) k_{\perp}^{2}\right]^{1 / 2} v_{A} \\
\omega_{i} \approx-\sqrt{2 \pi} k_{\perp} v_{t h \|} \frac{\beta_{\perp}^{2}}{\beta_{\|}^{2}} \frac{k_{\perp}}{k_{\|}} \exp \left[-\left(\frac{\sigma}{\beta_{\|}}+\frac{\left(1+\beta_{\perp}\right)}{\beta_{\|}} \frac{k_{\perp}^{2}}{k_{\|}^{2}}\right)\right]
\end{array}
$$

Even in a high- $\beta$ plasma the damping is relatively weak and is negligible for $k_{\perp}>k_{\|}$. The diamagnetic drift modifies the fast magnetosonic wave dispersion relation so that

$$
\left(\omega-\mathrm{k} \cdot \mathrm{V}_{0}\right)^{2}=\sigma k_{\|}^{2} v_{A}^{2}+\left(1+\beta_{\perp}-\frac{\beta_{\perp}^{2}}{\beta_{\|}} \frac{\tilde{\omega}_{*}}{\left(\omega-\mathrm{k} \cdot \mathrm{V}_{0}\right)}\right) k_{\perp}^{2} v_{A}^{2}
$$


(the Landau damping terms are exponentially small in this approximation) and the magnetosonic wave is coupled with a drift wave characterized by the frequency, $\tilde{\omega}_{*}=\sqrt{2} k_{\|} v_{t h \|}\left(\zeta_{*}^{(1)}+\right.$ $\left.\zeta_{*}^{(2)}+\zeta_{*}^{(3)}\right)$. If the drift frequency is small, coupling between the magnetosonic wave and drift wave is small and there is a slight modification shift in the real frequency of the mode.

For low frequency limit $\left(\left(\omega-\mathrm{k} \cdot \mathrm{V}_{0}\right), \omega_{*}<k_{\|} v_{t h i}\right.$ and $\left.\left(\omega / k_{\perp} v_{A}\right)^{2}<\omega / k_{\|} v_{t h i}\right)$ the kinetic mirror mode dispersion relation is given by

$$
\omega=\mathbf{k} \cdot \mathbf{V}_{0}+\hat{\omega}_{*}-i k_{\|} v_{t h \|} \sqrt{\frac{2}{\pi}} \frac{T_{\|}}{\beta_{\perp} T_{\perp}}\left(\frac{\sigma k_{\|}^{2}}{k_{\perp}^{2}}+\tau_{M H D}\right)
$$

where $\hat{\omega}_{*}=\left(3 \omega_{* p}-\omega_{* n}\right) / 2$ with $\omega_{* n}=-\rho_{i} v_{t h \perp} \mathbf{b} \times \mathbf{k} \cdot \nabla n / n$ and $\omega_{* p}=-\rho_{i} v_{t h \perp} \mathbf{b} \times \mathbf{k} \cdot \nabla P / P$. The first two terms in the dispersion relation are real frequency modification due to Doppler shift and diamagnetic drift respectively. The last term is the linear growth rate for the mirror mode. Without plasma flow and temperature gradient, the dispersion relation reduces to the drift mirror mode given by Hasegawa [24]. Notice that the stability criterion remains the same as for fluid theory, however unlike fluid theory the growth rate scales with $k_{\|}$.

\section{GLOBAL ANALYSIS}

We solve for the global mirror modes of the outlined system of equations for a steady state configuration based upon a typical pass through the magnetosheath and magnetopause $[6,17]$. In Fig. 1 we display typical steady state radial profiles for northward IMF. Although a true steady state configuration requires at least two dimensions in order to properly model the normal gradients in the plasma flow, the normal component of the bulk flow in the magnetosheath is typically much smaller than the plasma and magnetic pressures and thus does not appreciably affect the gradients that enter though the plasma pressure and magnetic field and thus the essential MHD profiles do satisfy a steady state solution to first order in $\rho V_{0}^{2} /\left(P+B^{2} / 2\right)$. On the other hand, the Doppler effects may be reasonably modeled by specifying a small normal velocity profile which is meant to be similar to what one would expect from a two dimensional steady state configuration. We introduce two spatial scales: 


\section{Equilibrium Profile}
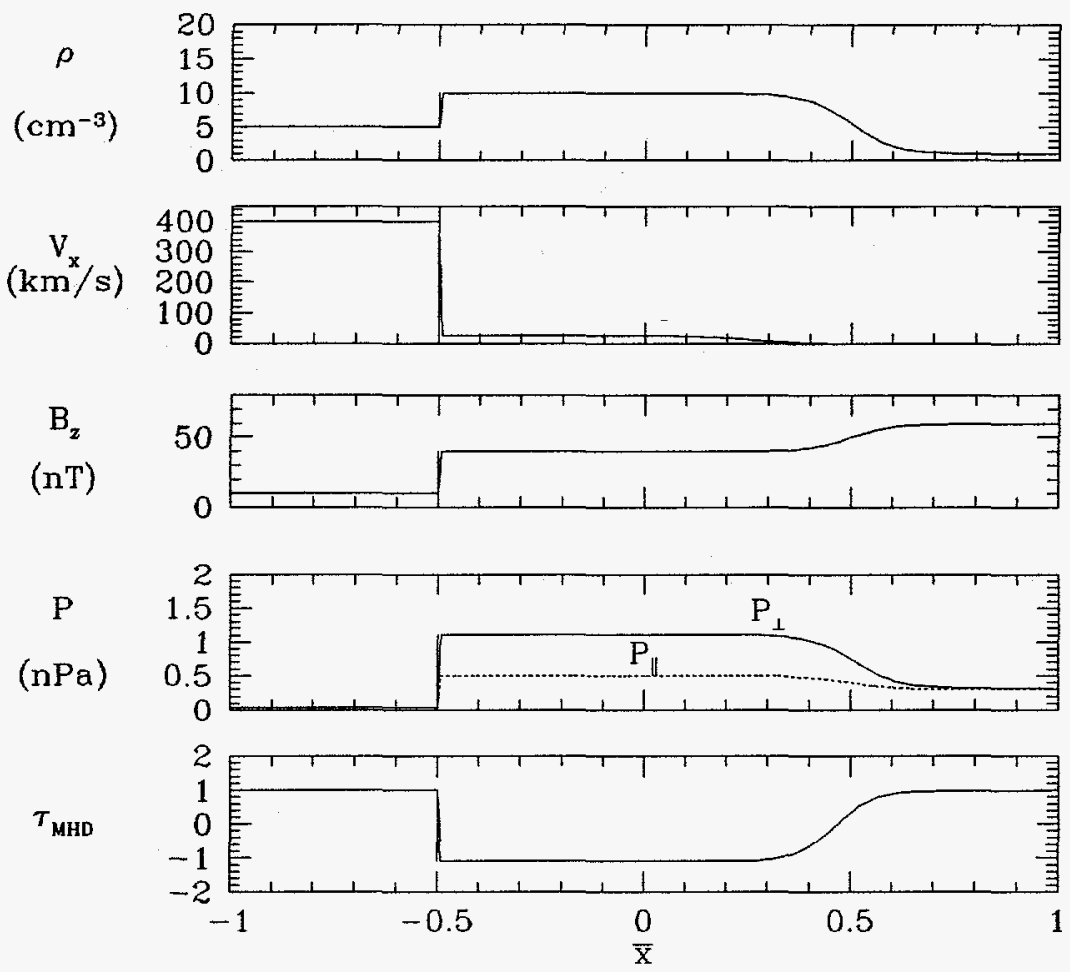

FIG. 1. Model steady state configuration for the global mode analysis for $\Delta=L_{M P} / L_{\tau}=0.05$ and $M_{A}=V_{0} / v_{A 0}=0.1$ where $v_{A 0}$ is the Alfvén velocity at $\bar{x}=0 . L_{M P}$ and $L_{\tau}$ are the width of the magnetopause and magnetosheath respectively.

$L_{\tau}$ is the scale length of the unstable region based upon $\tau_{M H D}<0$ which corresponds roughly to the width of the magnetosheath $\left(\approx 2-5 R_{E}[28]\right)$, and $L_{M P}$ represents the gradient scale length of the magnetopause $\left(\approx 10 \rho_{i}\right)$. The Mach number, $M_{A}$ is given by the ratio $V_{0} / v_{A 0}$ where $v_{A 0}$ is the Alfvén velocity at $\bar{x}=0$. The profile of $V_{0}$ is chosen to decrease from the bow shock slowly to a value of zero at the magnetopause. The dimensionless radial coordinate is $\bar{x}=\pi x / L_{\tau}$. The bow shock is at $\bar{x}=-0.5$ and magnetopause at $\bar{x}=0.5$ so that the width of the magnetosheath is $L_{\tau} / \pi$. The dimensionless frequency is $\bar{\omega}=\omega L_{\tau} / \pi v_{A 0}$. For typical parameters, this gives a frequency $f=(10-100) \bar{\omega} \mathrm{mHz}$. The wavevectors in the directions perpendicular to $x$ remain constant. They are defined by $k$ and $\theta$ where $k^{2}=k_{y}^{2}+k_{z}^{2}$ and $\theta=\tan ^{-1}\left(k_{y} / k_{z}\right)$. The results displayed here have $k_{0} \pi / L_{\tau}=5$ and $\theta=30^{\circ}$. For larger values of $\theta$, the mode becomes more stable. 
For a sharp magnetopause, there is a spectrum of purely growing modes up to an accumulation point. The boundary conditions limit the growth rate. For a smooth variance in the density, pressure and its anisotropy, and magnetic field, the kinetic part of $\tau$ has both a real and an imaginary part (which arises from the diamagnetic drift). The imaginary part of $\tau$ causes the eigenvalue to become complex rather than purely imaginary. If $\omega_{*}$ were uniform the real frequency is identically $\omega_{*}$, but for nonuniform diamagnetic effects, the real frequency arises as an averaged $\omega_{*}$. As the scale of the magnetopause is increased, the real frequency increases. The normal bulk plasma flow velocity also provides a significant contribution to the real frequency which is roughly given by an averaged $\mathbf{k} \cdot \mathbf{V}_{\mathbf{0}}$. (Note that we have not included the $\hat{y}$ component of flow which would lead to a further Doppler shift in the real frequency.)

To illustrate these two effects we plot in Fig. 2 the evolution of the mode frequencies as functions of the magnetopause gradient scale and the plasma flow, $\mathbf{V}_{\mathbf{0}}$. Each curve in this figure corresponds to an eigenfunction with a specific number of nodes beginning with the damped fundamental in ascending order. In particular, we plot the evolution of the $n=2,5,8,10,15$ modes, where $n$ is the number of nodes in the radial wave function $\left(k_{x} \sim(n+1) \pi / L_{\tau}\right)$. The solid curves show the evolution of the mode frequency as a function of $\Delta=L_{M P} / L_{\tau}$ with $\mathrm{V}_{0}=0$. The dashed curves show the evolution of modes as a function of $M_{A}$ with $\Delta=0.05$. Specific values of $\Delta$ and $M_{A}$ are indicated by symbols described in Table 1.

As expected, for small $L_{M P}$, the modes all line up on the imaginary axis. However, as $L_{M P}$ increases to more realistic values, the eigenvalues undergo excursions into the complex plane and are characterized by a significant real frequency. For substantial plasma flow $\left(M_{A}>0.1\right)$ the higher $n$ modes are suppressed for $n>8$. The lower $n$ pick up a substantial real frequency contribution from the Doppler shift with a roughly linear dependence, $\omega \sim V_{0}$.

In Fig. 3 we illustrate three radial wave structures of the $n=5$ eigenmode for three different plasma flow velocity and magnetopause boundary layer thickness. $\bar{x}=-0.5$ corresponds to the bow shock position and $\bar{x}=0.5$ the magnetopause position. The dotted and dashed 


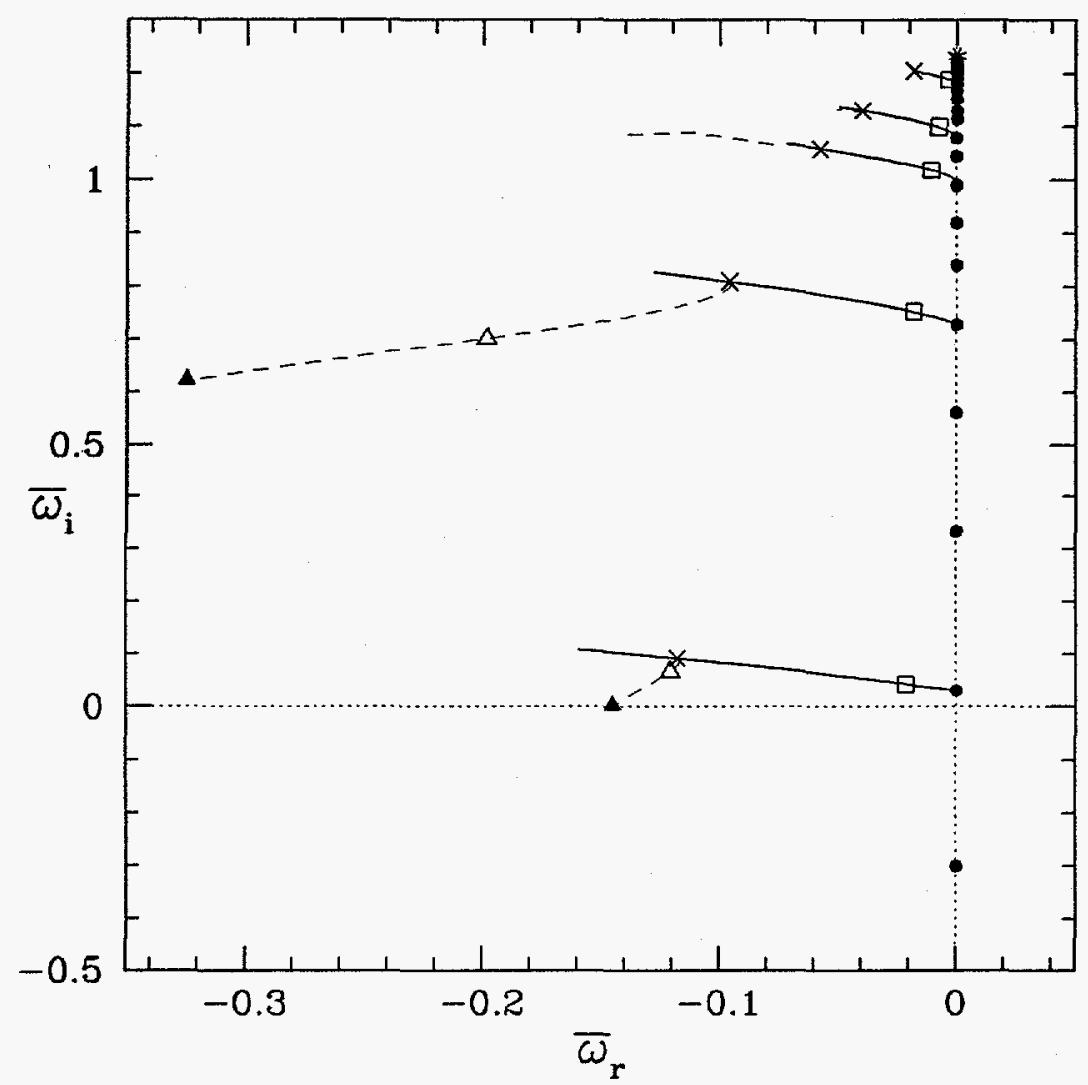

FIG. 2. Evolution of the eigenfrequencies $\left(\bar{\omega}=\omega L_{\tau} / \pi v_{A 0}\right)$ of the eigenfunctions. The solid lines correspond to evolution of the roots in $\Delta$ with $M_{A}=0$. The dashed lines correspond to evolution of the roots in $M_{A}$ with $\Delta=0.05$. Various points on these curves are indicated by symbols described in Table 1.

lines correspond to the real and imaginary part of the eigenfunctions of $\delta B_{\|}$, respectively. In all of these three cases, the real frequency is negative (same sign as diamagnetic drift frequency). This means that if the real part of the eigenfunction leads the imaginary part, then the mode travels to the left (phase velocity is negative). Conversely if the imaginary part leads the real part, the wave travels to the right.

For a sharp magnetopause with zero layer width $\left(\Delta \rightarrow 0, V_{0}=0, \bar{\omega}=(0,0.73)\right.$, the eigenfunction (the top panel in Fig. 3) is a standing wave. However, for a more realistic magnetopause gradient $\left(\Delta=0.05, M_{A}=0, \bar{\omega}=(-0.1,0.81)\right)$, the eigenfunction (the middle panel in Fig. 3) is complex and the real and imaginary parts of the eigenfunction are not in phase. Near the magnetopause where $\tau_{M H D}$ is small, the imaginary part of $\tau$ dominates over 


\begin{tabular}{||c|r|r||}
\hline \hline Symbol & $\Delta$ & $M_{A}$ \\
\hline$\bullet$ & 0 & 0 \\
$\square$ & 0.01 & 0 \\
$\times$ & 0.05 & 0 \\
$\Delta$ & 0.05 & 0.1 \\
$\Delta$ & 0.05 & 0.2 \\
\hline \hline
\end{tabular}

TABLE I. Specific values of $\Delta$ and $M_{A}$ are indicated by these symbols in Fig. 2.

the real part and leads to a phase shift in the real and imaginary parts of the eigenfunction. Because there is a real frequency associated with the wave, the structure slowly propagates as it grows. The wave amplitude is largest near the magnetopause. To understand why the eigenmode is skewed we consider the local dispersion relation in the magnetosheath region far away from the magnetopause boundary. The mode has largest growth with $k_{\|}>k_{y}$ and $\omega<k_{\|} V_{A}$ so that $\tau k_{x}^{2} \approx-\sigma k_{\|}$. Because diamagnetic drift effects are unimportant and $\omega_{r} \ll \omega_{i},\left|\tau_{r}\right| \gg\left|\tau_{i}\right|$ and we have $k_{x} \approx \pm k_{0}(1+i \delta)$ where $k_{0}=\sqrt{-\sigma k_{\|}^{2} / \tau_{r}}$ and $\delta=-\tau_{i} / 2 \tau_{r}$. For unstable modes with $\left|\omega / k_{\|} v_{t h}\right| \ll 1, \tau_{r}<0$ and $\tau_{i} \approx-\sqrt{\pi}\left(\beta_{\perp} T_{\perp} / T_{\|}\right) \omega_{r} / k_{\|} v_{t h}$. For $\omega_{r}<0, \tau_{i}>0$ and $\delta>0$. By inspection of the eigenfunction, $k_{x r}<0$ (the imaginary part of the eigenfunction leads the real part) and hence the eigenfunction will have the spatial behavior, $\exp \left(-i k_{0} x+\delta k_{0} x\right)$, which increases in amplitude in the positive $x$ direction. Because the real frequency is a consequence of the diamagnetic drift at the magnetopause, this growth can be interpreted to result from coupling of the wave with the diamagnetic drift associated with the pressure gradient. The diamagnetic drift causes the waves to drift with phase velocity toward the magnetopause boundary. As a result, the waves pile up against the magnetopause and the wave amplitude is enhanced. Although the diamagnetic drift is only large near the magnetopause, its effects are globally transmitted throughout the entire spatial domain leading to a slowly propagating mirror mode with real frequency. 


\section{$\delta \mathrm{B}_{\|}$for the $\mathrm{n}=5$ Eigenmode}

$$
\begin{aligned}
& \Delta=0 \\
& M_{A}=0 \\
& \Delta=0.05 \\
& M_{A}=0 \\
& \Delta=0.05 \\
& M_{A}=0.1
\end{aligned}
$$
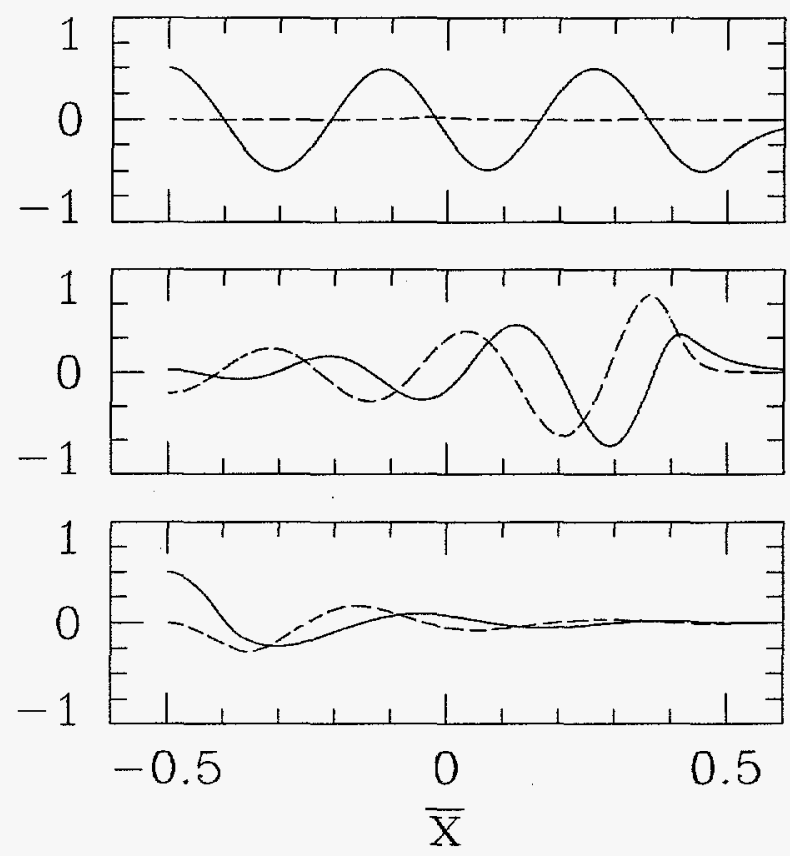

FIG. 3. Three radial wave structures of $\delta B_{\|}$(real: solid lines, imag: dashed lines) for the $n=5$ eigenmode for three different plasma flow velocity and magnetopause boundary layer thickness corresponding to three different points on the curve shown in Fig. 2. Notice that the density gradients and bulk flow strongly modify the mode structure. Note that $n$ indicates the number of nodes of the eigenfunction.

When the plasma flow is included $\left(\Delta=0.05, M_{A}=0.1, \bar{\omega}=(-0.2,0.7)\right)$ the real frequency of the mode is some combination of an averaged $\mathbf{k} \cdot \mathbf{V}_{0}$ and $\omega_{*}$. The real and imaginary parts of the eigenfunction (the bottom panel in Fig. 3) are out of phase which indicates that the wave phase velocity is in the flow direction. The solutions are skewed toward the bow shock. To physically understand the origin of the relatively larger real frequency, we note that the mode is most unstable when the arguments of the $Z$-functions $\left(\omega-\mathbf{k} \cdot V_{0}\right)$ is small so that $\omega-\mathbf{k} \cdot \mathbf{V}_{0} \simeq 0$ and the wave has a real frequency close to an average value of $\mathbf{k} \cdot \mathbf{V}_{\mathbf{0}}$. The behavior of the amplitude can be understood by considering $\left|\left(\omega-\mathbf{k} \cdot \mathbf{V}_{0}\right) / k_{\|} v_{t h}\right| \ll 1$. In this case $\tau_{i} \approx-\sqrt{\pi}\left(\beta_{\perp} T_{\perp} / T_{\|}\right)\left(\omega_{r}-\mathrm{k} \cdot \mathrm{V}_{0}\right) / k_{\|} v_{t h}$. The real part of the $\mathrm{n}=5$ mode local 
wavevector varies between -5 and -10 over most of the domain so that $\mathrm{k} \cdot \mathrm{V}_{\mathrm{o}} / \omega_{r} \approx-(2.5-5)$ whereas $\bar{\omega}_{r}=-0.2$ so that $\tau_{i}<0$. For unstable modes $\tau_{r}<0$, then $\delta=-\tau_{i} / 2 \tau_{r}<0$ and $k_{0}>0$. Hence the mode has a spatial behavior, $\exp \left(-i k_{0} x+\delta k_{0} x\right)$ so that the mode decays with increasing values of $x$. Physically, because the mirror mode propagates in the same direction as the flow but with a slower phase velocity, the mode will propagate toward the bow shock direction in the plasma moving frame. As the mode propagates toward the bow shock, its amplitude is enhanced by additional pressure anisotropy free energy and its energy accumulates near the the bow shock boundary because of the reflecting boundary condition there.

\section{DISCUSSION OF RESULTS}

Identification of observed compressional waves in the magnetosheath has been somewhat controversial. Indeed, many observations have found low frequency MHD waves with density and magnetic field out of phase. These waves have been interpreted as being either slow modes or mirror modes. Gleaves [2] observed compressional waves in the magnetosheath near the magnetopause and identified them as slow modes because those waves had a finite phase velocity toward the magnetopause in a different direction from the flow velocity. They dismissed those waves as being mirror modes based on the prediction of the uniform plasma theory that mirror modes are purely growing waves in the plasma rest frame so that they should be observed to propagate only with the flow direction in contrast to their observations. However, it is commonly believed that strong Landau damping of the slow mode in the magnetosheath inhibits its growth and therefore observations of waves with antiphase correlation between density and magnetic field were identified as drift mirror modes [7,9,29,30]. More recently, the work of Song et al. [8] and Denton et al. [9] addressed mode identification in the magnetosheath and near the magnetopause. The analysis of Denton et al. [9] identified the bulk of the observed waves near the magnetopause to be mirror modes. On the other hand, Song et al. [8] identified waves throughout the magnetosheath 
and found that while fast magnetosonic and mirror mode waves appear to be dominant in the middle magnetosheath, fast and slow magnetosonic waves are dominant near the magnetopause. In their argument based on uniform plasma theory the essential measurement that distinguishes between the mirror mode and slow magnetosonic waves is the so called Doppler ratio which is used to determine whether the wave has a finite phase velocity relative to the bulk plasma flow. Using two satellites they were able to determine that compressional waves observed just upstream of the magnetopause have antiphase relationship between magnetic field and density. Because they stand in the flow and, therefore, have a finite phase velocity in the frame of the flow, they were identified as slow modes.

The one-dimensional hybrid simulation of Omidi et al. [10] provides some insight as to a possible resolution of this controversy. In that simulation, a fast shock wave propagates away from a rotational discontinuity. Mirror mode waves arise in the wake of the shock due to pressure anisotropy and are carried against the rotational discontinuity by plasma flow. Near the region where the magnetic field increases, large amplitude waves appear to stand between the rotational discontinuity and the region of low plasma- $\beta$ referred to as a magnetic boundary. The waves appeared to stand in the flow and had finite real frequency relative to the plasma flow. Omidi et al. [10] called the waves MIAOW (mirror and slow mode) although it appears that the waves are simply compressional mirror modes that pile up at the magnetopause boundary. In the simulation the MIAOW waves were found to only occur for finite normal magnetic field. Indeed, as stated in our Local Theory Section, the mirror mode exists only for $\omega<k_{\|} v_{t h i}$, and if there were no normal magnetic field component $\mathbf{k} \cdot \mathbf{B}=0$, there is no instability.

Our calculations support the idea that compressional waves observed in the magnetosheath are global mirror modes. First, we demonstrated that diamagnetic drift effects resulting from a realistic magnetopause profile produces a significant real frequency in the global mirror mode with an increase in amplitude near the magnetopause boundary. Moreover, there is a finite real phase velocity directed toward the magnetopause. While the observations of Song et al. [8] found the compressional wave phase velocity directed away 
from the magnetopause, the observations of Gleaves et al. [2] seemed to indicate that occasionally the compressional waves do propagate toward the magnetopause. Secondly, when the plasma flow is introduced, the global mirror mode develops a phase velocity in the direction of the flow but is slower than the flow velocity. The result is that the wave would be observed to propagate toward the bow shock in the frame of the moving plasma and would have a finite real frequency in the plasma frame. The results also would seem to indicate that mirror mode wave activity should be stronger in the middle magnetosheath than near the magnetopause boundary which is consistent with observations by Song et al. [8]. The essential result of this work is that finite real frequency is associated with the global mirror mode due to nonlocal effects and the wave has a finite phase velocity relative to the plasma flow.

In general, the actual wave structure at the magnetopause will consist of a large number of modes because the growth rates for the modes are approximately the same (see Fig. 2). It is expected that finite Larmor radius effects will suppress the modes with sufficiently large $k_{x}$ and that there will be some range of $k_{x}$ with maximum growth. We expect that the observations will consist of many such modes with different $k_{x}$. As a result, there will be substantial beating between the waves leading to mirror mode wave structures with shorter spatial scale which would be comparable with the scales that are observed. The theory predicts a skew in the amplitude toward the magnetopause for small radial plasma flow and a skew toward the magnetosheath for large radial plasma flow. These are predictions from the theory that can be carefully compared with the observations in order to determine whether further refinements might be required for the theory.

The result of the one-dimensional hybrid simulation of Omidi et al. [10] differs from our analysis in a number of ways. Their analysis, for example, does not include the effect of diamagnetic drift because the wavevector, $\mathbf{k}$, is only along $\nabla(P, B, n)$. Moreover, the background model that they use is not in steady-state and therefore has time-dependent boundary conditions. It seems that the real frequency of the MIAOW waves results from the plasma flow effect because there is no diamagnetic drift effects in the simulation. 
Our one-dimensional analysis can still be improved by including coupling between the compressional and transverse components of the perturbed magnetic field which becomes essential near the shear Alfvén and slow magnetosonic resonances. If the flow velocity is large and/or the diamagnetic drift is small, Eq. (4) contains a singularity where $\tau(x)=M_{A}^{2}$. For an isotropic plasma, this singularity occur at $M_{A}=1$ and results because compressional waves cannot propagate against bulk flow which exceeds the Alfvén velocity. On the other hand, in anisotropic plasmas with no flow, the same singularity occurs where $\tau=0$ which corresponds to the transition between a region unstable to mirror mode and a region stable to mirror mode such as at the plasma depletion layer. If the density and pressure gradients are sufficiently large, the singularity is resolved by diamagentic drift effects. Moreover, in anisotropic plasmas this singularity also corresponds to the slow magnetosonic resonance condition. Therefore, it is likely that coupling to the sound wave will be important near that location. If the flow speed is sufficiently large, the resonance location will be shifted upstream. When the flow speed becomes too large no solution is found when $\tau=M_{A}^{2}$ because the diamagnetic effects no longer resolve the singularity. Resolution of the singularity involves coupling to the transverse magnetic field that we have neglected. In our future work we will include full coupling to the transverse magnetic field which resolves the singularity.

\section{SUMMARY}

In this paper we have presented an eigenmode analysis of the global mirror mode at the magnetopause using the kinetic-MHD model which accounts for both kinetic effects and global effects due to background gradients. The analysis demonstrates that:

- magnetopause gradients and plasma flow lead to a substantial real part of the eigenfrequency for the global mirror modes which is on the order of a combination of the Doppler shift frequency associated with plasma flow and diamagnetic drift frequencies,

- pressure and density gradients at the magnetopause modify the wave structure because of diamagnetic drift effects. The real frequency associated with the diamagnetic drift 
frequency provides a wave phase velocity in the direction of the magnetopause so that wave energy accumulates against the magnetopause boundary and the amplitude is skewed in that direction.

- plasma flow also gives rise to a real phase velocity, but the phase velocity is smaller than the flow velocity. As a result, the wave amplitude is increased in the wake of the plasma flow and piles up against the bow shock boundary and is skewed in that direction.

- boundary conditions are important because they impose restrictions that determine the global solution far away from the boundary and determine how the waves propagate within the region of instability,

- the kinetic-MHD formalism is useful when it is important to consider both global scale gradients and kinetic effects.

One obvious direction for future consideration is an extension to obtain mirror mode solutions based on a two-dimensional magnetosheath-magnetopause configuration which includes the two-dimensional plasma flow and the effects of curvature. Another important area for further consideration is coupling between the compressional and transverse components of the perturbed magnetic field which becomes essential near the shear Alfvén and slow magnetosonic resonances. Because these modes have a real frequency, they can couple strongly with the kinetic Alfvén wave near the location where the real frequency matches the local shear Alfvén resonance frequency which will occur if the background magnetic field rotates. Such coupling could lead to enhanced particle transport across the magnetopause boundary layer.

\section{APPENDIX: INTEGRALS OVER PRODUCTS OF BESSEL FUNCTIONS}

In the kinetic-MHD equations, the fields are multiplied by $J_{0}$ and $J_{1}$ so that integrals over the product $J_{0} J_{1}$ are frequently encountered. Integrals of this type may be evaluated 
through differentiation on the well known integral 6.633.1 from [31]

$$
\mathcal{I}_{1}(\alpha, \lambda) \equiv \int_{0}^{\infty} \exp \left(-\alpha \xi^{2}\right)\left[J_{0}(\lambda \xi)\right]^{2} \xi d \xi=\frac{1}{2 \alpha} \exp \left(-\lambda^{2} / 2 \alpha\right) I_{0}\left(\lambda^{2} / 2 \alpha\right)
$$

where $I_{0}$ is the modified Bessel function.

Kinetic effects for the core plasma involve two integrals

$$
\begin{aligned}
& \mathcal{I}_{2} \equiv \int_{0}^{\infty} \exp \left(-\xi^{2}\right) J_{0}(\lambda \xi) J_{1}(\lambda \xi) \xi^{2} d \xi \\
& \mathcal{I}_{3} \equiv \int_{0}^{\infty} \exp \left(-\xi^{2}\right) J_{0}(\lambda \xi) J_{1}(\lambda \xi) \xi^{4} d \xi \\
& \mathcal{I}_{5} \equiv \int_{0}^{\infty} \exp \left(-\xi^{2}\right) J_{0}(\lambda \xi) J_{1}(\lambda \xi) \xi^{6} d \xi
\end{aligned}
$$

Using the relationship

$$
\frac{\partial J_{0}(z)}{\partial z}=-J_{1}(z)
$$

we find that

$$
\begin{aligned}
& \mathcal{I}_{2}=-\left.\frac{1}{2} \frac{\partial \mathcal{I}_{1}}{\partial \lambda}\right|_{\alpha=1} \\
& \mathcal{I}_{3}=\left.\frac{1}{2} \frac{\partial^{2} \mathcal{I}_{1}}{\partial \lambda \partial \alpha}\right|_{\alpha=1} \\
& \mathcal{I}_{5}=-\left.\frac{1}{2} \frac{\partial^{3} \mathcal{I}_{1}}{\partial \lambda \partial \alpha^{2}}\right|_{\alpha=1} .
\end{aligned}
$$

We find through differentiation using the rule

$$
2 \frac{\partial I_{n}(z)}{\partial z}=I_{n-1}(z)+I_{n+1}(z)
$$

and

$$
\frac{2 n}{z} I_{n}(z)=I_{n-1}(z)-I_{n+1}(z)
$$

and with the auxiliary definition

$$
\Gamma_{n}(z)=\exp (-z) I_{n}(z)
$$

that 


$$
\begin{aligned}
& \mathcal{I}_{2}=\frac{\lambda}{4}\left[\Gamma_{0}\left(\lambda^{2} / 2\right)-\Gamma_{1}\left(\lambda^{2} / 2\right)\right] \\
& \mathcal{I}_{3}=\frac{\lambda}{2}\left\{\left[1-\lambda^{2} / 2\right] \Gamma_{0}-\left[1-\lambda^{2}\right] \Gamma_{1} / 2\right\} \\
& \mathcal{I}_{5}=\frac{3 \lambda}{2}\left\{\left[1-11 \lambda^{2} / 12+\lambda^{4} / 6\right] \Gamma_{0}-\left[1-9 \lambda^{2} / 4+\lambda^{4} / 2\right] \Gamma_{1} / 3\right\}
\end{aligned}
$$

The limiting forms of these expressions are of interest. The power series representations (in $\lambda$ ) of these functions is

$$
\begin{aligned}
& \mathcal{I}_{2}=\frac{\lambda}{4}\left(1-3 \lambda^{2} / 4+5 \lambda^{4} / 16+\ldots\right. \\
& \mathcal{I}_{3}=\frac{\lambda}{2}\left(1-9 \lambda^{2} / 8+5 \lambda^{4} / 8+\ldots\right. \\
& \mathcal{I}_{5}=\frac{3 \lambda}{2}\left(1-3 \lambda^{2} / 2+25 \lambda^{4} / 24+\ldots\right.
\end{aligned}
$$

The asymptotic expansions for large $\lambda$ are

$$
\begin{aligned}
\mathcal{I}_{2} & \sim \frac{1}{4 \sqrt{\pi} \lambda^{2}} \\
\mathcal{I}_{3} & \sim-\frac{7}{8 \sqrt{\pi} \lambda^{2}} \\
\mathcal{I}_{5} & \sim \frac{3}{16 \sqrt{\pi} \lambda^{2}}
\end{aligned}
$$

\section{ACKNOWLEDGMENTS}

This work is supported by the DoE Contract No. DE-AC02-76-CHO3073 and NSF grant ATM-9523331. 


\section{REFERENCES}

[1] C. P. Price, D. W. Swift, and L. C. Lee, J. Geophys. Res. 91, 101 (1986).

[2] D. G. Gleaves and D. J. Southwood, J. Geophys. Res. 96, 129 (1991).

[3] S. P. Gary, J. Geophys. Res. 97, 8519 (1992).

[4] P. Song, C. T. Russell, and M. F. Thomsen, J. Geophys. Res. 97, 8295 (1992).

[5] P. Song, C. T. Russell, and C. Y. Huang, J. Geophys. Res. 98, 5907 (1993).

[6] P. Song et al., J. Geophys. Res. 98, 187 (1993).

[7] B. J. Anderson, S. A. Fuselier, S. P. Gary, and R. E. Denton, J. Geophys. Res. 99, 5877 (1994).

[8] P. Song, C. T. Russell, and S. P. Gary, J. Geophys. Res. 99, 6011 (1994).

[9] R. E. Denton et al., J. Geophys. Res, 100, 5665 (1995).

[10] N. Omidi and D. Winske, J. Geophys. Res. 100, 11,935 (1995).

[11] S. P. Gary, M. E. McKean, and D. Winske, J. Geophys. Res. 98, 3963 (1993).

[12] A. Hasegawa and K. Mima, Phys. Rev. Lett. 36, 1362 (1978).

[13] L. C. Lee, J. R. Johnson, and Z. W. Ma, J. Geophys. Res. 99, 17405 (1994).

[14] J. R. Johnson and C. Z. Cheng, EOS Trans. AGU 76, 516 (1995).

[15] J. R. Johnson and C. Z. Cheng, EOS Trans. AGU 76, 252 (1995).

[16] B. J. Anderson, S. A. Fuselier, and D. Murr, Geophys. Res. Lett. 18, 1955 (1991).

[17] G. Paschmann, W. Baumjohann, N. Sckopke, and T. D. Phan, J. Geophys. Res. 98, $13,409(1993)$.

[18] S. P. Gary, S. A. Fuselier, and B. J. Anderson, J. Geophys. Res. 98, 1481 (1993). 
[19] S. P. Gary et al., Geophys. Res. Lett. 20, 1767 (1993).

[20] S. P. Gary and D. Winske, J. Geophys. Res. 98, 9171 (1993).

[21] D. Winske and N. Omidi, J. Geophys. Res. 100, 11,923 (1995).

[22] C. Z. Cheng, J. Geophys. Res. 96, 21,159 (1991).

[23] C. Z. Cheng and J. R. Johnson, in Physics of Space Plasmas (1995) (Scientific Publishers, Inc., ADDRESS, 1996), in press.

[24] A. Hasegawa, Phys. Fluids 12, 2642 (1969).

[25] D. J. Southwood and M. G. Kivelson, J. Geophys. Res. 98, 9181 (1993).

[26] E. A. Frieman and L. Chen, Phys. Fluids 25, 502 (1982).

[27] L. Chen and A. Hasegawa, J. Geophys. Res. 79, 1024 (1974).

[28] D. H. Fairfield, J. Geophys. Res. 75, 6700 (1971).

[29] B. T. Tsurutani et al., J. Geophys. Res. 87, 6060 (1982).

[30] D. Hubert, C. C. Harvey, and C. T. Russell, J. Geophys. Res. 94, 17305 (1989).

[31] I. S. Gradshteyn and I. M. Ryzhik, Table of Integrals, Series and Products (Academic Press, London, 1963). 